\title{
Determination of potential distribution in a three-barrier structure
}

\author{
D.M. Yodgorova, L.X. Zoirova, A.V. Karimov \\ Physical-Technical Institute of the Scientific Association "Physics-Sun" of the Academy of Sciences of the Republic of \\ Uzbekistan, Mavlanov str., 2B, 700084, Tashkent \\ Phone: +998-71-1331271,fax: +998-71-1354291; e-mail: karimov@uzsci.net
}

\begin{abstract}
Model $m_{1}-p-n-m_{2}$ structures with three barriers were considered; construction and technology of manufacturing the three-barrier $m_{1}-p$ AlGaInAs- $n \mathrm{GaAs}-m_{2}$ structure are presented. Experimental methods to determine voltages across every junction of the three-barrier structure were proposed. The mechanism of current transport when changing the blocked $p-n$ junctions and Schottky barriers were explained. It was shown that, at both polarities of operating regime, current characteristics are determined by blocked junctions. The obtained results are of interest for research of properties of threebarrier and similar phototransistor structures in response to external influences.
\end{abstract}

Keywords: three-barrier structure, metal-semiconductor, $p$ - $n$ junction.

Manuscript received 07.03.06; accepted for publication 23.10.06.

\section{Introduction}

Nowdays, improvement of semiconductor device effectiveness is carried out in the technological process using several barriers. Then effectiveness of structures of solar cell type is improved using series connection of several cells switched in one direction with tunnel junctions between them in one cascade [1]. In the photodiode structure approaching, screw planar structures with two Shottky barriers are formed to improve their speed [2]. Existence of space charge layers near two barriers causes a decrease in the total capacity of the structure and as a result the speed increase takes place in them. In the diode structures, all processes are determined by a blocked junction due to one of the barriers is always switched in the opposite direction of the others, besides the voltage across the structure is determined as a whole by the blocked contact. That is why, their current-voltage characteristics (CVC) possess inverse brunches.

Two-side sensitive two-barrier $m-p-m, \quad m-n-m$ structures with the Shottky barriers were described in [3]; owning to both rectifying metal-semiconductor junctions in them the photodetectors have high photosensitivity in the shortwaved spectral range when exciting them from any side.

To improve photosensitivity and speed of the photodiode structure in the work [4], integration of $p-n$ junction and reverse-based metal-semiconductor junctions into one aggregate structure was carried out. So, two-side sensitive two-barrier $m-p-n[5,6]$ and three-barrier $m-p-n$ $m$ structures [7] were obtained. Their parameters depend essentially on technology of formation of active layers and potential barriers. Identity of CVC brunches was determined by perfection and correlation of carrier concentrations in regions forming the rectifying junction $[8,9]$. Specimens of the above mentioned works had rather more high photosensitivity than an ordinar photodiode structure with one junction. In $[10,11]$, processes of photoelectric amplification and formation of spectral characteristics in three-barrier $m_{1}-p$ AlGaInAs$n \mathrm{GaAs}-m_{2}$ structure were researched [12]. In operating regime when changing the applied voltage, they were redistributed between reverse-based barriers. The character of their redistribution and process of growth determines their photoelectric characteristics. Until recently theoretical [13] and experimental [14] approaches to researching the symmetrical $m-n-m$ and nonsymmetrical two-barrier structures are known.

In this work, described was redistribution of potential in symmetrical three-barrier hetero- $m_{1}$ $p$ AlGaInAs- $n$ GaAs- $m_{2}$-structures.

\section{Experimental samples and methods of research}

To investigate the potential redistribution between barriers and determine the factors determining processes of current characteristics forming, special three-barrier heterostructures with symmetrical output characteristics were manufactured. $n \mathrm{GaAs}: \mathrm{O}$ with the carrier concentration $N_{m}=1 \cdot 10^{15} \mathrm{~cm}^{-3}$ was chosen as a substrate. Heterojunctions were obtained by growth of $\mathrm{Zn}$-doped pAlGaInAs with $N_{p}=2 \cdot 10^{16} \mathrm{~cm}^{-3}$ on GaAs substrate. Thickness of the films was 2 to $3 \mu \mathrm{m}$. Then diode $m_{1}-p-n$ $m_{2}$ structure was formed depositing gold onto 
$p \mathrm{Al}_{0.08} \mathrm{Ga}_{0.82} \mathrm{In}_{0.1} \mathrm{As}$ and silver with the thickness $\sim 70 \AA$ onto the back surface of $n \mathrm{GaAs}: \mathrm{O}$ in vacuum. Here $\varphi_{1}^{m_{1}-p}=0.42 \mathrm{eV}$ and $\varphi_{2}^{n-m_{2}}=0.88 \mathrm{eV}$ for metalsemiconductor junctions $p \mathrm{Al}_{0.08} \mathrm{Ga}_{0.82} \mathrm{In}_{0.1} \mathrm{As}$ and $n \mathrm{GaAs}-$ Ag, accordingly. Heights of potential barriers $\left(\varphi_{1}^{m_{1}-p}\right.$ and $\varphi_{2}^{n-m_{2}}$ ) were determined from the dependence of square root of photoresponse, recalculated per one photon energy. That is in accordance with the data of [15]. The area of the structure was reduced to $5 \times 5 \mathrm{~mm}$ by scribing. In the three-barrier structure, $m_{1}-p$ and $n-m_{2}$ junctions were series-connected but $p-n$ junction is approaching. This structure is two-side sensitive because both its surfaces are photodetectors, photocurrent is created by lighting any side.

At forward bias of $p-n$ junction, two metalsemiconductor junctions work within regime of reverse bias, moreover the current in circuit is limited only by the current of forward-biased metal-semiconductor junction with the bigger resistance. At reverse bias of $p-n$ junction, a current is determined by blocked $p-n$ junction and forward-biased metal-semiconductor junctions connected as one (Fig. 1). As shown in the equivalent scheme (Fig. 1), the resistances of the first and third junctions are Shottky barrier connected in series, and it is possible to consider them as one resistance $R_{1}^{1}=R_{1}+R_{3}$, and the resistance of $p$ - $n$ junction as $R_{2}$. From slops of curves for forward and reverse biases (Fig. 2) it is clear that the resistance $R_{1}^{1}=R_{2}$, and three-barrier structure is symmetrical. We can determine the voltage across every junction from the data of CVC of Fig. 2 using the methods [14] for a twobarrier structure.

We designate the voltage across $R_{1}^{1}$ as $V_{1}^{1}$ and $R_{2}$ as $V_{2}$. The sum of these voltages is the total voltage:

$V_{\text {tot }}^{m_{1}-p-n-m_{2}}=V_{1}^{1}+V_{2}^{p-n}$.

In their turn

$$
V_{1}^{1}=V_{1}^{m_{1}-p}+V_{3}^{n-m_{2}}
$$

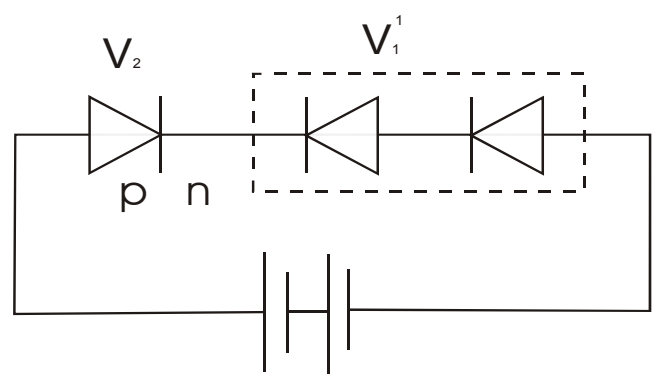

Fig. 1. Simplified equivalent scheme of a three-barrier structure reduced to the two-barrier one. where $V_{1}^{m_{1}-p}$ and $V_{3}^{n-m_{2}}$ are the voltages across $m_{1}-p$ and $n-m_{2}$ junctions. Because of the equality of currents flowing through all the junctions:

$R_{1}^{1} V_{2}^{p-n}=V_{1}^{1} R_{2}^{p-n}$

then

$V_{2}^{p-n}=\frac{R_{2}^{p-n} V_{1}^{1}}{R_{1}^{1}}$ and

$V_{2}^{p-n}=R_{2}^{p-n} I_{\text {tot }}^{m_{1}-p-n-m_{2}}$

For the three-barrier $m_{1}-p-n-m_{2}$ system, assuming $R_{2}^{p-n}=R_{1}^{1}=R_{1}^{m_{1}-p}=R_{3}^{n-m_{2}}$ as it is in symmetrical structure, we determine the resistance of the separate ( $R_{2}$ or $R_{1}^{1}$ ) junction in the point of contrary bend of CVC:

$R_{2}=\frac{R_{0}}{2}=\frac{V_{0}}{2 I_{0}}$,

where $V_{0}, I_{0}$ are the voltage and current in the point of contrary bend, respectively. Resulting resistance of three $p-n$ junctions connected in series is equal:

$R_{0}=\frac{V_{0}}{I_{0}}$.

Because [14]

$V_{2}^{p-n}=\frac{V_{0}}{2 I_{0}} I_{\text {tot }}^{m_{1}-p-n-m_{2}}$

and

$V_{1}^{1}=V_{\text {tot }}^{m_{1}-p-n-m_{2}}-V_{2}^{p-n}$

on the base of the formulas (8) and (9) in the case of blocked metal-semiconductor junctions, we can calculate the voltages across $R_{2}^{p-n}$ and $R_{1}^{1}$ junctions. When

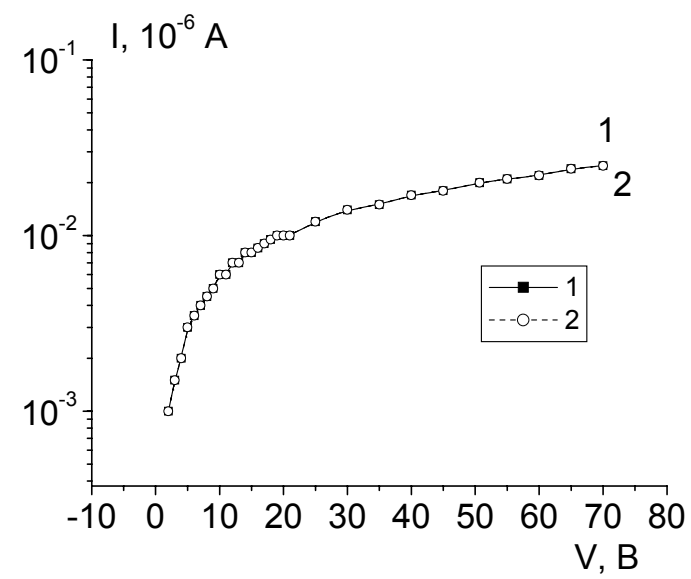

Fig. 2. Current-voltage characteristic of three-barrier $m_{1}$ - $p$ AlGaInAs- $n$ GaAs- $m_{2}$ structure within the regimes of forward (1) and reverse (2) biases of heterojunction. 
changing the polarity of the applied voltage, the data for $R_{2}^{p-n}$ junction must be vary by the same way as for $R_{1}^{1}$ one. In the case when the carrier concentrations in $p$ and $n$ regions are equal and the heights of metalsemiconductor potential barriers are equal, too, $\varphi_{1}=\varphi_{2}$, we have

$$
\begin{aligned}
& V_{1}^{m_{1}-p}=V_{3}^{n-m_{2}}, \\
& V_{1}^{1}=2 V_{1}^{m_{1}-p} .
\end{aligned}
$$

As a result, from the dependence (8) taking into account

$V_{1}^{m_{1}-p}=V_{1}^{1} / 2$,

we obtain formula for the voltage across the metalsemiconductor $m-p$ junction

$V_{1}^{m_{1}-p}=\frac{V_{0}}{2 I_{0}} \frac{I_{\text {tot }}^{m_{1}-p-n-m_{2}}}{2}$.

We also find expressions for the voltages

$V_{1}^{1}=V_{1}^{m_{1}-p}+V_{3}^{n-m_{2}}$,

$V_{3}^{n-m_{2}}=V_{1}^{1}-V_{1}^{m_{1}-p}$

In the case when the heights of potential barriers are not equal $\left(\varphi_{1} \neq \varphi_{2}\right)$, voltages across them can be different, and there is certain correlation between their resistances

$\frac{R_{3}^{n-m_{2}}}{R_{1}^{m_{1}-p}}=\frac{E_{3}^{0}}{E_{1}^{0}}$

where $E_{3}^{0}$ is the electric field of $m_{1}-p$ junction, which is determined by relation of the potential barrier height to the thickness of the space charge layer [16]

$$
E_{3}^{0}=\frac{\varphi_{3}}{W_{3}^{0}}=\varphi_{3}^{n-m_{2}} /\left(\frac{2 \varepsilon \varepsilon_{0}}{q} \frac{\varphi_{1}^{n-m_{2}}}{N_{n}}\right)^{1 / 2} .
$$

Here $E_{1}^{0}$ is the electric field of $m_{1}-p$ junction, it is equal

$$
E_{1}^{0}=\frac{\varphi_{1}}{W_{1}^{0}}=\varphi_{1}^{n-m_{2}} /\left(\frac{2 \varepsilon \varepsilon_{0}}{q} \frac{\varphi_{1}^{m_{1}-p}}{N_{p}}\right)^{1 / 2}
$$

Within the regime of forward-biased $p$ - $n$ junction, taking into account (16)-(18) and solving the equations (2) and (18)

$$
\frac{V_{3}^{n-m_{2}}}{V_{1}^{m_{1}-p}}=\frac{E_{3}^{0}}{E_{1}^{0}}
$$

we obtain expression relating the voltage across the $m_{1}-p$ junction with the total voltage

$$
V_{1(\mathrm{rev})}^{m_{1}-p}=V_{1(\mathrm{rev})}^{1} \frac{E_{1}^{0}}{E_{1}^{0}+E_{3}^{0}} .
$$

Here, $\frac{E_{1}^{0}}{E_{1}^{0}+E_{3}^{0}}$ is the coefficient describing relation of electrical fields between metal-semiconductor barriers connected in series, besides

$\left(\frac{E_{1}^{0}}{E_{1}^{0}+E_{3}^{0}}\right)_{\text {forw }}=\left(\frac{E_{1}^{0}}{E_{1}^{0}+E_{3}^{0}}\right)_{\mathrm{rev}}$

Using the formula (2), we determine the voltage across another $n-m_{2}$ junction

$V_{3(\text { rev })}^{n-m_{2}}=V_{1(\text { rev })}^{1}-V_{1(\text { rev })}^{m_{1}-p}$.

Within the blocked $p-n$ junction regime, we find the voltage across the forward-biased $m_{1}-p$ junction by the same way

$V_{1 \text { (forw ) }}^{m_{1}-p}=V_{1 \text { (forw ) }}^{1} \cdot \frac{E_{1}^{0}}{E_{1}^{0}+E_{3}^{0}}$

and using the formula (23) we determine the voltages across the second forward-biased $n-m_{2}$ junction

$V_{3 \text { (forw ) }}^{n-m_{2}}=V_{1 \text { (forw) }}^{1}-V_{1 \text { (forw) }}^{m_{1}-p}$.

Then, we find $V_{2}^{p-n}$ voltage across the blocked $p-n$ junction

$V V_{2(\mathrm{rev})}^{p-n}=V_{\text {tot(rev) }}^{\mathrm{m}_{1}-p-n-m_{2}}-V_{1 \text { (forw) }}^{1}$.

So, we have the formulas to determine the voltages across every junction of the three-barrier structure.

\section{Results and discussion}

In researching Au- $p \mathrm{Al}_{0.08} \mathrm{Ga}_{0.82} \mathrm{In}_{0.1} \mathrm{As}-n \mathrm{GaAs}-\mathrm{Ag}$ structure, because of resemblance of CVC when varying the applied voltage, it is enough to estimate the applied voltages $V_{2}^{p-n}$ and $V_{1}^{1}$ for only one of the regimes and then to replace the obtained values for the other regime.

In the point of contrary bend at $V=5 \mathrm{~V}, I=$ $0.003 \mu \mathrm{A}$ (Fig. 2). Then taking into account the calculated values of the coefficient and formula (12) and using the formulas (16), (17), (19), (21), we determine the voltages across the $p-n$ heterojunction: $V_{2 \text { (forw) }}^{p-n}$ and $V_{1(\mathrm{rev})}^{1}-$ across the metal-semiconductor seriesconnected junctions as well as $V_{1}^{m_{1}-p}$ and $V_{3}^{n-m_{2}}-$ across the separate $m_{1}-p$ and $n-m_{2}$ junctions.

Within the forward-biased $p-n$ heterojunction regime, we calculated values of the space charge layer thickness, electrical field intensity, capacities of blocked $m_{1}-p$ and $n-m_{2}$ junctions using the formulas (26)-(29)

$W^{m_{1}-p}=\left(\frac{2 \varepsilon \varepsilon_{0}}{q} \frac{\left(\varphi_{1}^{m_{1}-p}+V_{1(\mathrm{rev})}^{m_{1}-p}\right)}{N_{p}}\right)^{1 / 2}$, 
Table 1. Data of symmetrical $A u-p \mathrm{Al}_{0.08} \mathrm{Ga}_{0.82} \mathrm{In}_{0.1} \mathrm{As}-n \mathrm{GaAs}-\mathrm{Ag}$ structure within the forward-biased $p$-n heterojunction regime.

\begin{tabular}{|l|c|c|c|c|c|c|c|c|}
\hline$V_{\text {tot }}^{m_{1}-p-n-m_{2}}, \mathrm{~V}$ & 2 & 5 & 10 & 15 & 20 & 30 & 40 & 70 \\
\hline$I_{\text {tot }}^{m_{1}-p-n-m_{2}}, \mu \mathrm{A}$ & 0.001 & 0.003 & 0.006 & 0.008 & 0.010 & 0.014 & 0.017 & 0.025 \\
\hline$V_{2 \text { (forw) }}^{p-n}, \mathrm{~V}$ & 1 & 3 & 6 & 8 & 10 & 14 & 17 & 25 \\
\hline$V_{1 \text { (rev) }}^{1}, \mathrm{~V}$ & 1 & 2 & 4 & 7 & 10 & 16 & 23 & 45 \\
\hline$V_{1 \text { (rev) }}^{m_{1}-p}, \mathrm{~V}$ & 0.758 & 1.56 & 3.0 & 5.3 & 7.58 & 12.12 & 17.43 & 34.1 \\
\hline$W^{m_{1}-p}, 10^{-5} \mathrm{~cm}$ & 2.72 & 3.53 & 4.64 & 6 & 7.1 & 8.89 & 10.6 & 14.7 \\
\hline$E, 10^{5} \mathrm{~V} / \mathrm{cm}$ & 0.43 & 0.56 & 0.74 & 0.95 & 1.13 & 1.41 & 1.67 & 2.35 \\
\hline$C, 10^{9} \mathrm{~F}$ & 11.58 & 8.92 & 6.79 & 5.25 & 4.44 & 3.54 & 2.97 & 2.14 \\
\hline$V_{3 \text { (rev) }}^{n-m_{2}}, \mathrm{~V}$ & 0.242 & 0.484 & 1.0 & 1.7 & 2.42 & 3.88 & 5.57 & 10.89 \\
\hline$W^{n-m_{2}}, 10^{-5} \mathrm{~cm}$ & 8.53 & 10.37 & 14.3 & 19.6 & 25.1 & 36.2 & 49.02 & 89.5 \\
\hline$E, 10^{5} \mathrm{~V} / \mathrm{cm}$ & 0.13 & 0.13 & 0.13 & 0.13 & 0.13 & 0.13 & 0.13 & 0.13 \\
\hline$C, 10^{-9} \mathrm{~F}$ & 2.85 & 2.4 & 1.7 & 1.24 & 0.97 & 0.67 & 0.49 & 0.28 \\
\hline
\end{tabular}

Table 2. Data of symmetrical $A u-p \mathrm{Al}_{0.08} \mathrm{Ga}_{0.82} \mathrm{In}_{0.1} \mathrm{As}-\mathrm{nGaAs}-\mathrm{Ag}$ structure within the reverse-biased $p$-n heterojunction regime.

\begin{tabular}{|l|c|c|c|c|c|c|c|c|}
\hline$V_{\text {tot }}^{m_{1}-p-n-m_{2}}, \mathrm{~V}$ & 2 & 5 & 10 & 15 & 20 & 30 & 40 & 70 \\
\hline $\begin{array}{c}m_{1}-p-n-m_{2} \\
I_{\text {tot }}\end{array}$ & 0.001 & 0.003 & 0.006 & 0.008 & 0.010 & 0.014 & 0.017 & 0.025 \\
\hline$V_{1 \text { (forw) }}^{1}, \mathrm{~V}$ & 1 & 3 & 6 & 8 & 10 & 14 & 17 & 25 \\
\hline$V_{\begin{array}{l}m_{1}-p \\
1 \text { (forw) }\end{array}, \mathrm{V}}^{n-m_{2}}, \mathrm{~V}$ & 0.758 & 2.27 & 4.55 & 6.06 & 7.58 & 10.61 & 12.88 & 18.95 \\
\hline$V_{3 \text { (forw) }}^{n-n}, \mathrm{~V}$ & 0.242 & 0.73 & 1.45 & 1.94 & 2.42 & 3.39 & 4.12 & 6.05 \\
\hline$V_{2 \text { (rev) }}^{p}$ & 1 & 2 & 4 & 7 & 10 & 16 & 23 & 45 \\
\hline$W^{p-n}, 10^{-5} \mathrm{~cm}$ & 16.46 & 20.08 & 25.8 & 32.6 & 38.2 & 47.45 & 56.36 & 77.98 \\
\hline$E, 10^{5} \mathrm{~V} / \mathrm{cm}$ & 0.12 & 0.15 & 0.196 & 0.25 & 0.29 & 0.36 & 0.43 & 0.59 \\
\hline$C, 10^{-9} \mathrm{~F}$ & 1.91 & 1.57 & 1.22 & 0.97 & 0.82 & 0.66 & 0.56 & 0.40 \\
\hline
\end{tabular}

$W^{n-m_{2}}=\left(\frac{2 \varepsilon \varepsilon_{0}}{q} \frac{\left(\varphi_{1}^{n-m_{2}}+V_{3(\mathrm{rev})}^{n-m_{2}}\right.}{N_{n}}\right)^{1 / 2}$.

Capacity and electrical field intensities for every junction were calculated on the base of these space charge layer thickness values by using the formulas

$C=\frac{\varepsilon \varepsilon_{0} S}{W}$,

$E=\frac{V}{W}$.

Within the reverse-biased heterojunction regime, we calculated the value of the $p-n$ junction space charge layer thickness [13]

$$
W_{p-n}^{2}=\left[\frac{2 \varepsilon_{1} \varepsilon_{0} \varepsilon_{2} \varepsilon_{0} \cdot\left(N_{p}+N_{n}\right)^{2} \cdot\left(V_{D}-V_{2}^{p-n}\right)}{q \cdot\left(\varepsilon_{1} \varepsilon_{0} N_{n}+\varepsilon_{2} \varepsilon_{0} N_{p}\right) \cdot N_{n} N_{p}}\right],
$$

where $E_{1}=11.4$ for $\mathrm{Al}_{0.08} \mathrm{Ga}_{0.82} \mathrm{In}_{0.1}$ As and $E_{2}=11$ for GaAs; $V_{D}=1.054 \mathrm{~V}$. Electrical field intensities and blocked $p-n$ heterojunction capacity were calculated using the formulas (36), (37). The calculated data are summarized in Tables 1 and 2. Interconnection between index of $\mathrm{CVC}$, carrier concentration, space charge layer thickness, dielectrical permittivity, capacity and electrical fields of junctions are ascertained on the base of the data shown in Tables 1 and 2.

\section{Conclusion}

Considered are the model $m_{2}-p-n-m_{2}$ structures with three barriers, given are construction and manufacturing technology of three-barrier structure on the base of gallium arsenide. Proposed is the experimental calculation method to determine voltages that drop on each transitions of the three-barrier structure. Obtained results can be applied for research the properties of three-barrier and analogical phototransistors under external affect. 


\section{References}

1. J.I. Alferov, V.M. Andreyev, V.D. Rumyantcev, Tendency and perspective of solar photoenergetics development // Fizika, tekhnika poluprovodnikov 38(8), p. 937-947 (2004) (in Russian).

2. S.V. Averin, Determination of contact characteristics of metal-semiconductor for counterpintle photodiode structure // Pis'ma in ZhTF 16(4), p. 49-53 (1990) (in Russian).

3. D.M. Yodgorova, E.N. Yakubov, Detectors of optical signal on a base of Au-nSi-Al and $\mathrm{Au}-\mathrm{nSi}$ diodes // Tekhnologiya $i$ dizain $v$ elektronnoy apparature No 4, p. 39-42 (2005) (in Russian).

4. A.V. Karimov, Three-barrier photodiode of Karimov. Patent RUz \#933 from 15.04.1994.

5. A.V. Karimov, D.M. Yodgorova, K. Vakhobov, A.G. Gaibov, Kh.T. Igamberdiyev, Bilateralsensitive photodiode structures in gallium arsenide and cadmium sulphite system // J. Eng. Phys. and Thermophys. 76(1), p. 167-169 (2003).

6. A.V. Karimov, D.M. Yodgorova, E.N. Yakubov, Research of structures corrugated photoreceiving surface // Semiconductor Physics, Quantum Electronics and Optoelectronics 7(4), p. 378-382 (2004).

7. A.V. Karimov, Three-barrier photodiode // Lazernaya tekhnika i optoelektronika No 3, p. 53-55 (1991) (in Russian).
8. A.P. Belyaev, V.P. Rubetc, Kh.A. Tashkhodjaev, I.P. Kalinkin, Inversion as conduction of zinc selenide in heterostructure // Fizika i tekhnika poluprovodnikov 27(3), p. 527-532 (1993) (in Russian).

9. A. Farenbuh, R. Byub, Solar elements. Energoatomizdat, Moscow, 1987, p. 84-90 (in Russian).

10. I.M. Vikulin, V.I. Stafeyev, Physics of semiconductor devices. Radio and svyaz', Moscow, 1990, p. 264 (in Russian).

11. A.V. Karimov, Photovoltage in three-barrier structure // Lazernaya tekhnika $i$ optoelektronika No 3, p. 83-85 (1993) (in Russian).

12. A.V. Karimov, D.A. Karimova, Three-junction $\mathrm{Au} / \mathrm{AlGaAs}(\mathrm{n}) / \mathrm{GaAs}(\mathrm{p}) / \mathrm{Ag}$ photodiode. Materials of Sci. Conf. in Semiconductor Processing. Vol. 6, Issues 1-3, February-June 2003, p. 137-142.

13. A. Milns, D. Foyht, Heterojunctions and junctions of metal-semiconductor. Mir, Moscow, 1975 (in Russian).

14. A.V. Karimov, D.M. Yodgorova. Determination of two-barrier photodiode structures characteristics with metal-semiconductor transitions // Tekhnologiya $i$ dizain $v$ elektronnoy apparature No 5, p. 27-30 (2005) (in Russian).

15. S.M. Czi, Physics of semiconductor devices. Book 1. Mir, Moscow, 1984, p. 286-306 (in Russian).

16. M. Shur, Modern devices on a base of gallium arsenide. Moscow, Mir, 1991 (in Russian). 